\title{
Deformed Microstructure and Hardness of Hadfield High Manganese Steel
}

\author{
Lihe Qian, Xiaoyong Feng and Fucheng Zhang* \\ State Key Laboratory of Metastable Materials Science and Technology, Yanshan University, Qinhuangdao 066004, P. R. China
}

\begin{abstract}
The distribution of micro/nano-indentation hardness and microstructures of deformed Hadfield high manganese steel have been investigated by means of micro/nano-indentation tests, and optical and electron microscopic observations. Indentation tests demonstrated that there exists a very non-uniform distribution of hardness, with a hardness variation of $\sim 30 \%$ in different grains and in different regions of the same grain. Microscopic observations indicated that the large non-uniformity of micro/nano-hardness is caused mainly by the underlying nonhomogeneous substructures (twins, dislocations, etc.), which are closely associated with the non-uniform work hardening in the deformed Hadfield steel, while the effects of the austenite grain boundary and the grain orientation of the indentation plane are not significant. [doi:10.2320/matertrans.M2011121]
\end{abstract}

(Received April 22, 2011; Accepted May 27, 2011; Published July 13, 2011)

Keywords: Hadfield high manganese steel, railway crossing, micro-indentation hardness, work hardening, deformation structure

\section{Introduction}

Hadfield high manganese steel, with a full austenite microstructure at room temperature, has been receiving much attention, and it is used in a variety of applications such as railway crossings, crawler treads for tractors and impact hammers, because of its excellent work-hardening rate, high toughness and high wear resistance. Many efforts have been made to study the deformation mechanisms of Hadfield steel, with an attempt to correlate the excellent work hardening properties of the steel with its evolved microstructures. ${ }^{1-5)}$ Deformation and work-hardening properties of Hadfield steel are often assessed by uniaxial tension or compression tests and also by hardness measurements; ${ }^{6,7)}$ such assessments are essentially averaged results of deformation over a whole specimen or over a relatively large volume of material. Nevertheless, microscopic observations revealed that the evolved substructures of the Hadfield steel subjected to deformation are mostly non-homogeneous, ${ }^{8}$ ) indicative of non-uniform deformation and work hardening that has occurred in the deformed steel.

The understanding of non-uniform deformation and work hardening of materials is of critical importance, especially in failure analysis of practical industrial products, since locally concentrated straining associated with non-uniform work hardening tends to initiate damage and cracking. ${ }^{9,10)}$ Some researchers have, indeed, evaluated the degree of straining heterogeneity due to mechanical twinning of Hadfield steel by a digital image correlation method; ${ }^{8)}$ however, not much attention has been paid to the local-scale hardness in deformed Hadfield steel. A non-uniform distribution of hardness on the local scale, which may be influenced by a number of factors such as grain boundary, grain orientation of the indentation plane as well as the microstructures under the indentation tip, ${ }^{11-13)}$ can be expected in the deformed Hadfield steel. The aim of the present work is to study the non-uniform work hardening of a practical deformed Hadfield steel crossing by characterizing the local-scale hardness distribution by means of Vickers and nano-

*Corresponding author, E-mail: zfc@ysu.edu.cn indentation hardness measurements, and to explore the origin of the hardness non-uniformity by investigating the local microstructural features in the deformed Hadfield steel by means of optical microscope, electron back-scattered diffraction (EBSD) and transmission electron microscope (TEM).

\section{Experimental}

The distribution of local-scale hardness and deformed microstructure in a Hadfield steel crossing, which had been subjected to deformation in railway use, was investigated. The chemical composition (mass\%) of the steel was: $1.2 \mathrm{C}$, $12.4 \mathrm{Mn}, 0.60 \mathrm{Si}, 0.016 \mathrm{~S}, 0.022 \mathrm{P}$ and the balance Fe. The Hadfield steel crossing had been solution treated and water quenched before putting into use. The mechanical properties of the Hadfield steel are shown in Table 1. When taken off the railway line, the crossing had undergone about 160 million-ton transportation load with a combination of repeated rolling, compression and impact forces from train wheels. Since deformation, work hardening, as well as microstructure evolution in Hadfield steel below the working surface of the crossing may vary with the distance from the surface, due to the difference in stress level at different depths,${ }^{7)}$ hardness and microstructure inspections were made at three different depths, i.e. $0.4,1.2$ and $2.0 \mathrm{~mm}$ below the crossing's surface. Specimens with the inspected surfaces parallel to the working surface were extracted from the crossing by electro-discharge machining. For comparison, the non-deformed Hadfield steel having the same chemical composition and in the same heat treated state was also examined.

After the specimens were carefully polished, micro hardnesses and their distribution were measured using a Vickers pyramid hardness tester with an applied load of $2 \mathrm{~N}$ and also using a nano-indentation tester with a Berkovich diamond indenter with an applied load of $3 \mathrm{mN}$. Metallographic microstructures were examined by optical microscopy (OM) after specimens were polished and etched with $4 \%$ Nital. More details about the microstructural features were further inspected using a scanning electron microscope 
Table 1 Mechanical properties of the investigated Hadfield steel.

\begin{tabular}{ccccc}
\hline $\begin{array}{c}\text { Elongation to } \\
\text { fracture, } \\
\delta / \%\end{array}$ & $\begin{array}{c}\text { Reduction } \\
\text { in area, } \\
\phi / \%\end{array}$ & $\begin{array}{c}\text { Ultimate tensile } \\
\text { strength, } \\
\sigma_{\mathrm{b}} / \mathrm{MPa}\end{array}$ & $\begin{array}{c}\text { Impact } \\
\text { toughness, } \\
\alpha_{\mathrm{k}} / \mathrm{J} . \mathrm{cm}^{-2}\end{array}$ & $\begin{array}{c}\text { Hardness, } \\
\mathrm{HV}\end{array}$ \\
\hline 35.8 & 22.9 & 840 & 320 & 225 \\
\hline
\end{tabular}

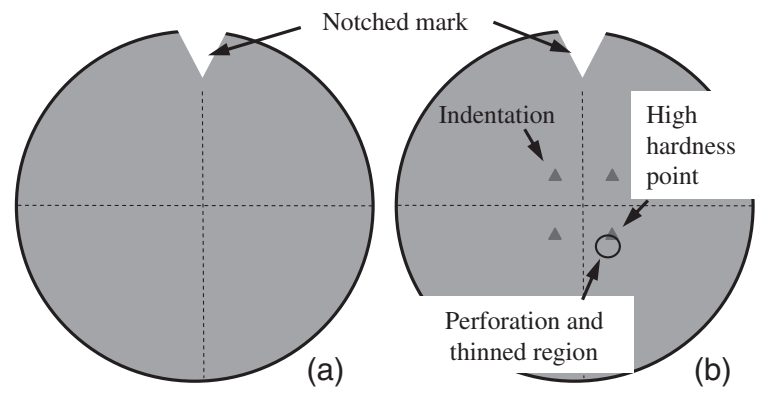

Fig. 1 A schematic showing (a) a notch mark cut at the edge of the disk sample and (b) nano-indentations made in the central region of the sample for determining the location of the perforated regions. Dashed lines show approximately the alignment lines under the indentation microscope.

(SEM, KYKY2800-B), and a scanning electron microscope (SEM, Model LEO-1450) equipped with electron backscattered diffraction device (EBSD, HKL-EBSD system). Specimens for EBSD analysis were mechanically ground and then chemically polished. EBSD scans were performed with a scanning step size of $5 \mu \mathrm{m}$.

Substructures of specimens were examined with a transmission electron microscope (TEM, Hitachi H-800) operating at $200 \mathrm{kV}$. In order to correlate the measured local-scale hardness values with the corresponding substructures, samples were carefully prepared for TEM observations. Slices of $0.4 \mathrm{~mm}$ thick were cut from the subsurface layer below the crossing's surface, and mechanically ground down to $30 \mu \mathrm{m}$ thick before disks of $3 \mathrm{~mm}$ in diameter were punched. A triangle notch as a mark was cut at the edge of each disk, as is schematically shown in Fig. 1(a). Four nano-indentations spaced $200 \mu \mathrm{m}$ were made in the central region of each disk with an applied load of $3 \mathrm{mN}$ (Fig. 1(b)). Sizes of the indentation pits were about $3 \mu \mathrm{m}$ in diameter and $2 \mu \mathrm{m}$ in depth, which were much smaller than the thickness of the disks. The disk samples were thinned to perforation using a Gatan precision ion polishing system. Multiple samples were perforated. Only those thin foiled samples with perforated holes nearby the indentation pits with an obviously higher hardness value or a lower hardness value measured (Fig. 1(b)) were chosen for TEM observations.

\section{Results and Discussion}

\subsection{Hardness distribution}

Vickers hardness tests were performed on the deformed Hadfield steel at three different depths below the crossing's surface. 64 measurements were made at each depth in a pattern of $8 \times 8$ matrix spaced $260 \mu \mathrm{m}$ on the plane parallel to the crossing's surface, with some indentations possibly being in the interior of grains and some others possibly being at grain boundaries. A typical pattern of the indentations at the depth of $0.4 \mathrm{~mm}$ is shown in Fig. 2(a). The micrograph was taken from the sample etched with $4 \%$ Nital subsequent to the indentation test, to reveal severe plastic deformation bands in the grains. The hardness values measured at three depths are shown in Fig. 2(b). Two features are evident. One is that the mean of the measured hardness values decreases slightly with increasing the depth. This is understandable considering that larger deformation occurred at locations closer to the crossing's surface, which generated increased wok hardening, when compared with deeper locations. Another feature to be especially noted is that there is a large scatter of $150 \sim 180 \mathrm{HV}$ (i.e. about one-third variation) for each depth group, with the averaged hardness values being $520 \sim 580 \mathrm{HV}$. This large scatter is remarkable, indicative of large non-uniform hardness distribution in the deformed Hadfield steel. Several factors, such as grain boundary, grain orientation of the indentation plane, and local substructure under the indentation tip, may be responsible for this observation. Grain boundaries are often reported to have higher strength and hardness than the interior of grains at room temperature, mostly in the case of non-deformed materials. ${ }^{14)}$ This is supposed to be true also in the present deformed steel. To check this supposition, the hardness data at the depth of $1.2 \mathrm{~mm}$ were divided into two parts, according to whether the Vickers indentation position was located at grain boundaries or in the interior of grains. However, the grouped data, as is shown in the inset of Fig. 2(b), indicate that the hardness values measured within only the interior of grains still show a large variation, suggesting that the strengthening effect of grain boundary is not the major reason for the large non-uniform hardness distribution in the present deformed steel.

Conversely, based on 64 hardness measurements on the non-deformed Hadfield steel, the hardness values were found to vary in the range of $215 \sim 230 \mathrm{HV}$, with a very small scatter of $\sim 15 \mathrm{HV}$. Since these measurements were performed in different grains (i.e. with different grain orientations of the indentation plane) or possibly at grain boundaries, it is apparent that the effects of grain boundaries and the grain orientation of the indentation plane on the measured hardness are ignorable. This is in agreement with the result on the hardness measurements of single-crystal copper in the literature, ${ }^{12)}$ where the variation of the hardness measured in a single-crystal copper did not exceed $6 \%$ when varying the orientation of the indentation plane.

To further exclude the possible effects of the grain boundary hardness and the grain orientation of the indentation plane, two or three Vickers indentations were performed inside same grains (thus with the same grain orientations of the indentation plane). Forty grains of the deformed and additional forty grains of the non-deformed Hadfield steel were respectively examined. Multiple measurements demonstrated that the deviation of the hardness values measured in the same grain of the non-deformed steel is negligibly small (Fig. 2(c)); however, large deviations were observed in most of the deformed grains (Fig. 2(d)). To further rule out possible effects from the Vickers indenter, nano-indentation tests with an applied load of $3 \mathrm{mN}$ were performed at the depth of $0.4 \mathrm{~mm}$ below the crossing's 


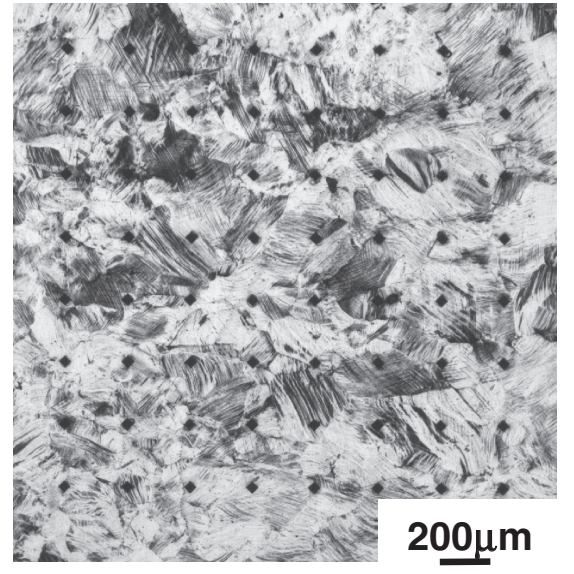

(a)

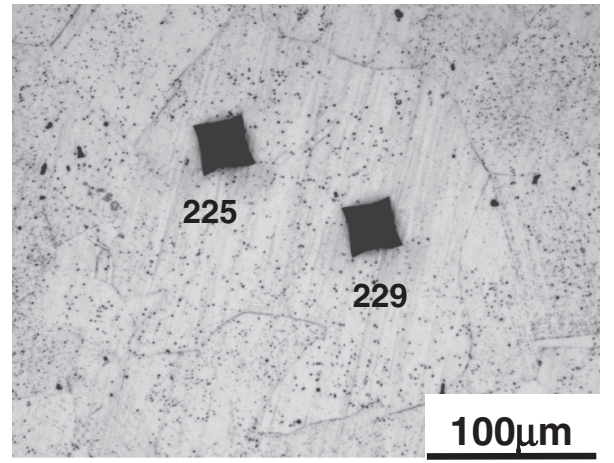

(c)

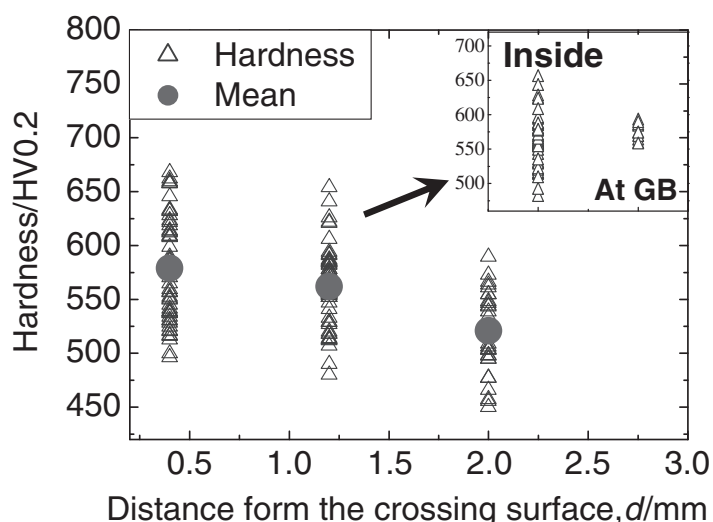

(b)

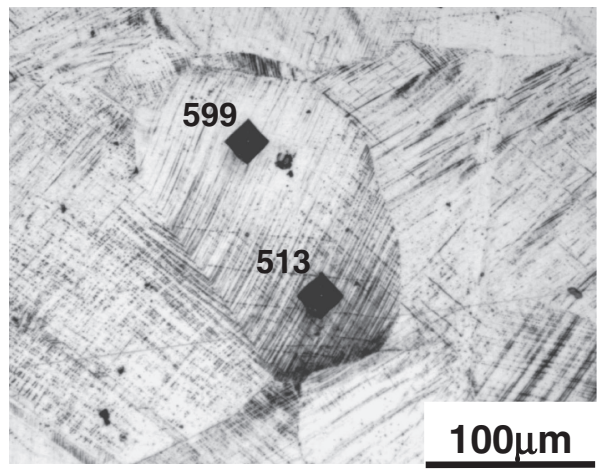

(d)

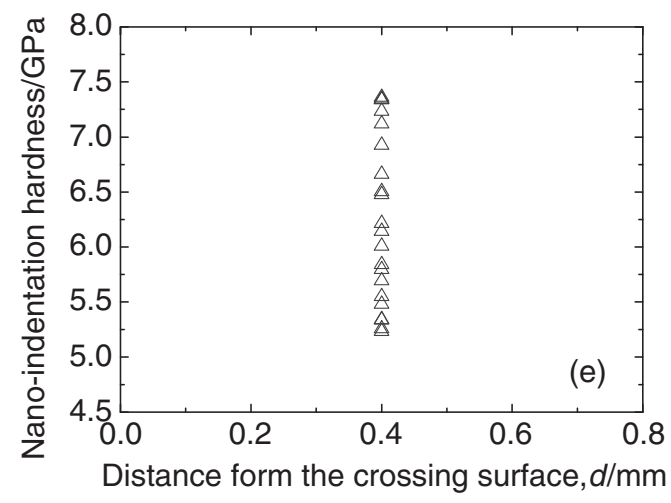

Fig. 2 Vickers indentation results: (a) indentation pattern of $8 \times 8$ matrix spaced $260 \mathrm{~mm}$ on the plane $0.4 \mathrm{~mm}$ deep; (b) measured hardnesses at the depths of $0.4,1.2$ and $2.0 \mathrm{~mm}$, where for the depth of $1.2 \mathrm{~mm}$ the hardness data inside the grains and at the GBs are separately shown in the inset; two Vickers indentations (the numbers indicate the hardness values) measured in a single grain of (c) the non-deformed and (d) deformed steel; (e) nanoindentation hardensses obtained at the depth of $0.4 \mathrm{~mm}$.

surface. The results are shown in Fig. 2(e). As expected, the hardness values obtained change between 5.2 and $7.4 \mathrm{GPa}$ with a similar variation to that by the Vickers indenter. These findings strongly suggest that the large variations in the measured hardness were not caused primarily by the austenite grain boundary and the grain orientation of the indentation plane, while the underlying non-homogeneous substructures may play a major role, as will be examined below.

\subsection{Deformed microstructure}

The metallographic microstructure of the non-deformed Hadfield steel is characterized by a single-phase austenite structure, and no deformation traces are seen after etching (Fig. 3(a)). The austenitic grain size is about $140 \mu \mathrm{m}$. The microstructure at the depth of $1.2 \mathrm{~mm}$ below the crossing's surface is shown in Fig. 3(b). Some austenitic grains are full of intense straight deformation bands, and some grains contain fewer or no deformation bands; however, this does not certainly mean that the grains without visible deformation bands were not plastically deformed, because the deformation traces may be embedded within the grains, parallel to the viewing surface. The appearance of deformation bands in the grains after etching indicates the surface relief caused by twinning, ${ }^{6}$ as will be confirmed by TEM observations. Under optical microscope, the grains with intense deformation bands look dark while the grains with fewer deformation bands look bright. The difference in color of grains results from the difference in the extent of etching of the grains, since grains with more deformation bands exposed outwards 

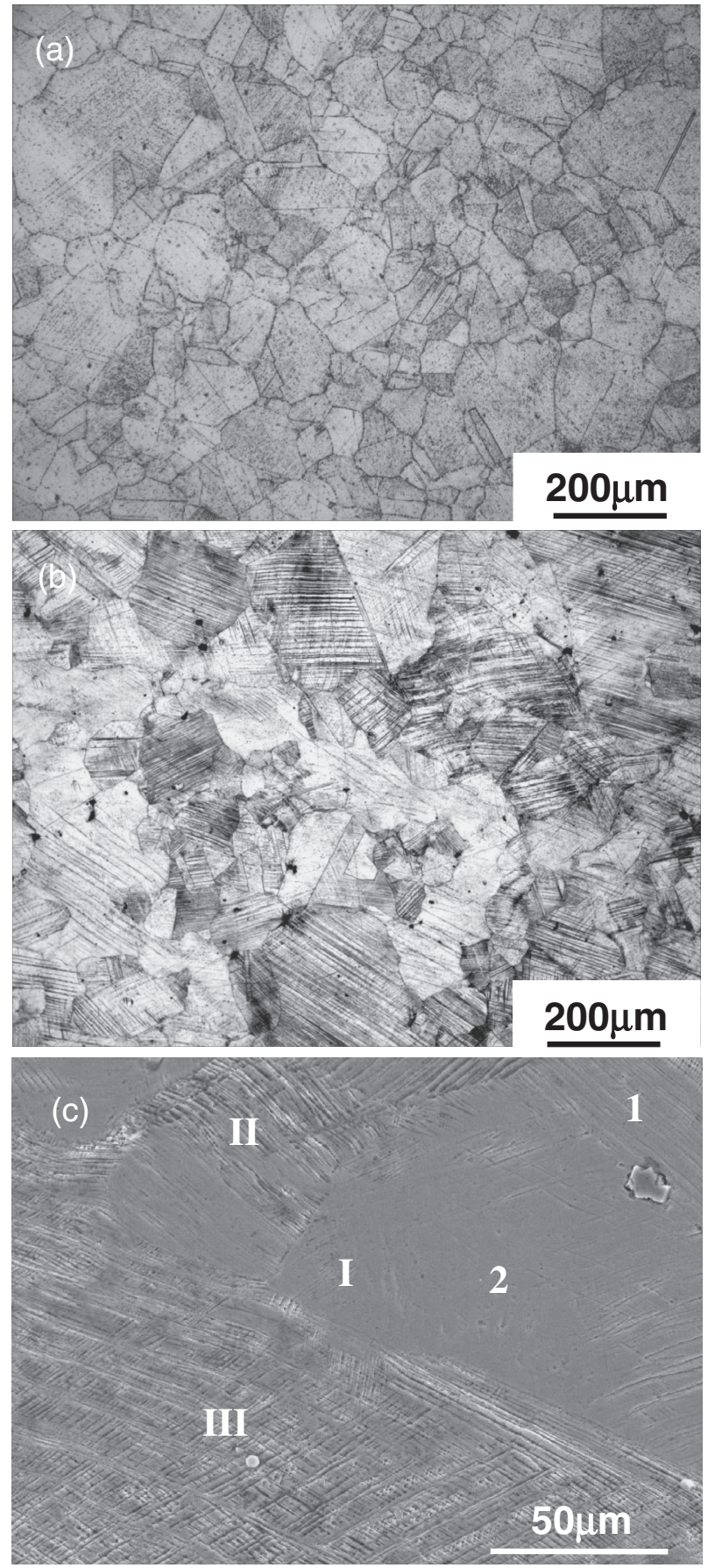

Fig. 3 (a) Optical micrograph of the non-deformed microstructure; (b) and (c) optical and SEM micrographs of the deformed microstructure at the depth of $1.2 \mathrm{~mm}$ below the crossing's surface.

tend to be more easily etched, thus showing deep color, and vise versa.

For more information on deformation bands, deeply etched specimens were examined in the SEM. As is shown in Fig. 3(c), several patterns of deformation bands are apparent. In Grain I, multiple parallel deformation bands pass through the grain in Area 1, whereas no deformation bands are seen in Area 2. In Grain II, parallel intense deformation bands prevail, whilst in Grain III two systems of intense deformation bands that are intersected with each other are clearly visible.

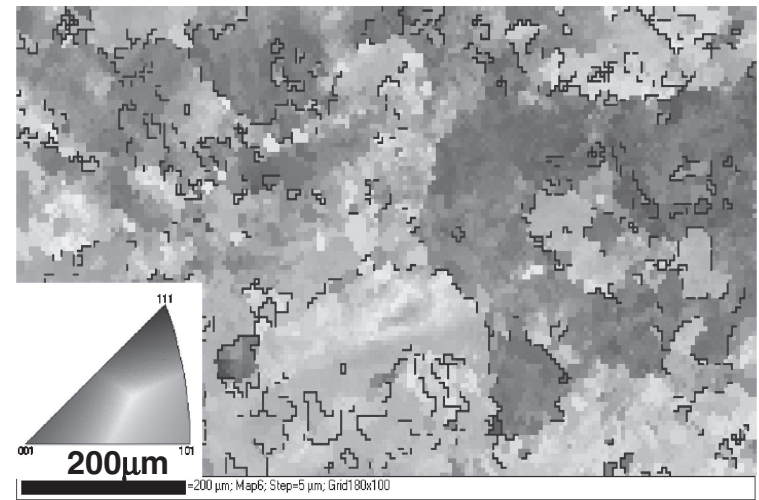

(a)

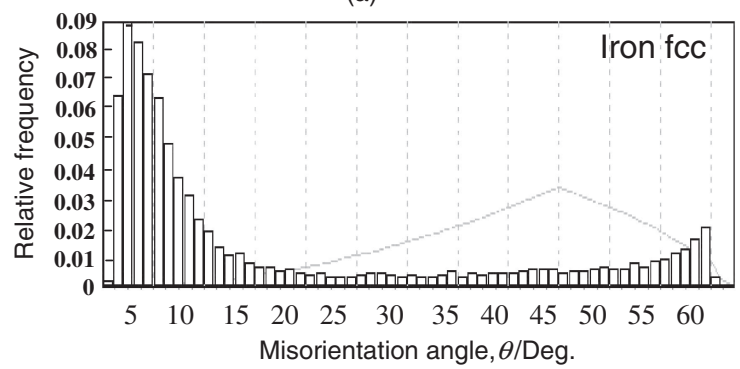

(b)

Fig. 4 (a) The orientation map and (b) the relative frequency distribution of misorientation angle of grain boundaries of the deformed microstructure at the depth of $1.2 \mathrm{~mm}$ below the crossing's surface.

The orientation map and the misorientation distribution of grain boundaries of the deformed microstructure at the depth of $1.2 \mathrm{~mm}$, obtained by EBSD technique, are shown in Fig. 4(a) and (b), respectively. Subgrains bounded by black lines are visible (Fig. 4(a)), which are much smaller than the normal austenite grains (Fig. 3). A significantly higher frequency of grain boundaries with misorientations of $2 \sim 15^{\circ}$ is visible (Fig. 4(b)). These low angle grain boundaries are believed to be associated with different dislocation substructures caused by slip. ${ }^{8)}$ Furthermore, a lower proportion of high angle $\left(55 \sim 60^{\circ}\right)$ boundaries are seen (Fig. 4(b)). These high angle boundaries correspond to austenite grain boundaries as well as the boundaries of twins typified by a misorientation angle of $55^{\circ}$ between the twins and their parent matrix. ${ }^{8)}$

Multiple TEM observations demonstrated that deformation twins are prevalent in the high-hardness samples of the deformed Hadfield steel. This is typically evident in the TEM micrographs of Fig. 5(a) and (b), which were taken in the same grain around the perforation with a high hardness of 7.0 GPa. A large area of parallel microtwins is visible, as confirmed by the selected area diffraction pattern in the inset (Fig. 5(a)). There exist heavily entangled dislocations within the microtwins and a plenty of dislocation pile-ups near/at the twin boundaries, whilst defect- or dislocationclear zones are notable in the matrix between the microtwins. Figure 5(b) shows intersecting twins with a high density of entangled dislocations in the twins, and some dislocation cells that are present in the untwined region. In contrast, deformation twins are seldom observable in the low-hardness samples. As is typically shown in Fig. 5(c) with a low hardness of $5.4 \mathrm{GPa}$ measured around the 


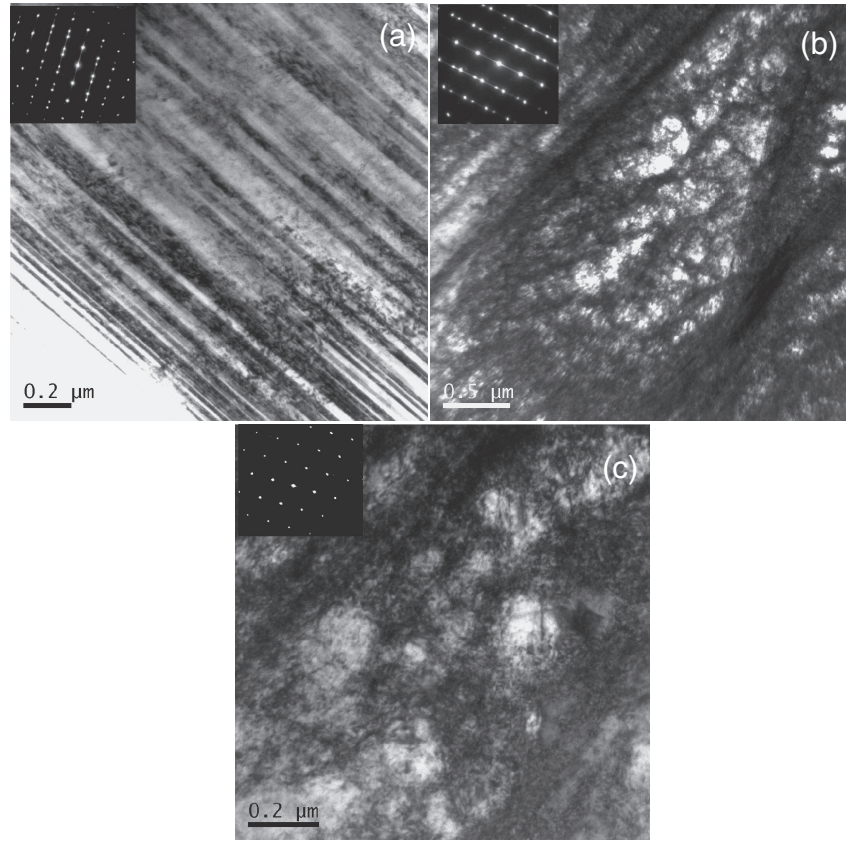

Fig. 5 TEM micrographs of the deformed microstructure at the depth of $1.2 \mathrm{~mm}$ below the crossing's surface: (a) and (b) in a high-hardness sample, and (c) in a low-hardness sample.

perforation, microscopic slip bands other than twins are frequently observed. These slip bands may be a result of planar slip due to the low stacking fault energy of the Hadfield steel, which are often formed at low strain levels. ${ }^{15)}$ It is found that the dislocation structures are very nonhomogeneous from one area to another. High densities of entangled dislocations are seen within the slip bands, while dislocation cell structures are evident between the bands.

The formation of different patterns of substructures in the deformed Hadfield steel is attributable to the difference in the degree of deformation occurring in different grains or in different regions of the same grain. Depending on the stress level and the orientation of each individual grain with respect to the loading direction, one arrangement of deformation twinning may prevail in one grain (Fig. 5(a), (b)), while dislocation slide may dominate plastic deformation in a nearby grain (Fig. 5(c)) ${ }^{16)}$ Even in the same grain, different patterns of substructures may also be generated (Fig. 5(a), (b)), because of different local constraints resulting from the GBs and the surrounding grains.

The hardness value measured on a local scale is dependent on the microstructure under the indentation tip. Nondeformed Hadfield steel possesses a homogeneous austenite microstructure, demonstrating a relatively uniform distribution of hardness. In contrast, as revealed by OM, SEM and TEM observations, various patterns of deformation bands or twin/dislocation substructures are present in different grains as well as in different regions of same grains of the deformed Hadfield steel. Multiple twins serve as strong barriers for dislocation motions, and high densities of dislocations pile up at twin boundaries (Fig. 5(a)), which leads to largely increased work hardening and enhanced hardness within the twinned region; however, in the untwined region, plastic deformation occurs primarily by dislocation glide, as evidenced by slip bands and dislocation cell structure (Fig. 5(c)), causing a moderate increase in both work hardening and hardness. Accordingly, with the deformed Hadfield steel containing non-uniform substructures of twins or dislocations in different local regions as the starting material for hardness measurement, a non-uniform distribution of hardness is anticipated. Such a non-uniform distribution of hardness due to the underlying non-uniform substructures has been known in cyclically deformed copper crystals, in which the cyclically saturated microstructure of copper is composed of a hard matrix (essentially vein dislocation structure) and a soft permanent slip band (ladderlike dislocation structures), with plastic deformation mainly localized within the permanent slip band. ${ }^{9)}$

A good understanding of the non-uniform distribution of microstructure and hardness in deformed Hadfield steel is of critical significance, since damage and cracking tends to be initiated at local severely strained regions because of the mismatch of deformation, as was reported in cyclically deformed copper where crack tends to be initiated at the boundary of the permanent slip band and matrix. ${ }^{9,10)}$ Further work is currently being undertaken to investigate crack initiation and the incompatibility of deformation owing to the non-uniform hardness distribution in the deformed Hadfield steel by means of in situ SEM observation and crystal plasticity finite element method.

\section{Conclusions}

The results of the above investigations can be summarized as follows. It has been demonstrated that there exists a very non-uniform distribution of micro/nano-indentation hardness, with a hardness variation of about $30 \%$ in different grains as well as in different regions of even the same grain of the deformed Hadfield high manganese steel. The large nonuniformity of hardness distribution in the deformed Hadfield steel is not caused mainly by the austenite grain boundary and grain orientation of the indentation plane, but by the underlying non-homogeneous substructures, with higher hardness in multiple-twin regions and lower hardness in dislocation-prevailing regions. Further work is suggested to investigate crack initiation and the incompatibility of deformation due to non-uniform hardness distribution in deformed Hadfield steel.

\section{Acknowledgements}

This work was supported by The National Natural Science Foundation of China (Grant Nos. 50925522, 50871094 and 50821001), and the Natural Science Foundation of HeBei Province (Grant Nos. E2009001632 and E2011203066).

\section{REFERENCES}

1) P. H. Adler, G. B. Olson and W. S. Owen: Metall. Trans. A 17 (1986) 1725-1737.

2) B. Hutchinson and N. Ridley: Scr. Mater. 55 (2006) 299-302.

3) M. Abbasi, S. Kheirandish, Y. Kharrazi and J. Hejazi: Mater. Sci. Eng. A 513-514 (2009) 72-76.

4) E. G. Astafurova, G. G. Zakharova and H. J. Maier: Scr. Mater. 63 (2010) 1189-1192. 
5) W. Owen and M. Grujicic: Acta Mater. 47 (1998) 111-126.

6) I. Karaman, H. Sehitoglu, K. Gall, Y. I. Chumlyakov and H. J. Maier: Acta Mater. 48 (2000) 1345-1359.

7) F. C. Zhang, Z. N. Yang, L. H. Qian, F. C. Liu, B. Lv and M. Zhang: Scr. Mater. 64 (2011) 560-563.

8) C. Efstathiou and H. Sehitoglu: Acta Mater. 58 (2010) 1479-1488.

9) Z. F. Zhang, Z. G. Wang and Z. M. Sun: Acta Mater. 49 (2001) 2875 2886.

10) B.-T. Ma and C. Laird: Acta Metall. 37 (1989) 325-336.

11) V. G. Gavriljuk, H. Berns, C. Escher, N. I. Glavatskaya, A. Sozinov and
Y. N. Petrov: Mater. Sci. Eng. A 271 (1999) 14-21.

12) J. Joost, W. D. Vlassak and J. Nix: Mech. Phys. Solids 42 (1994) 1223 1245 .

13) E. Bayraktar, F. A. Khalid and C. Levaillant: J. Mater. Proc. Tech. 147 (2004) 145-154.

14) S. Kobayashi, S. Tsurekawa and T. Watanabe: Acta Mater. 53 (2005) 1051-1057.

15) J. W. Christian and S. Mahajan: Prog. Mater. Sci. 39 (1995) 1-157.

16) J. A. Jimenez and G. Frommeyer: Mater. Charact. 61 (2010) 221226. 